\title{
Políticas Neoliberales y Primera Infancia: una Revisión Desde el Enfoque de Derechos y la Inclusión Educativa en Chile ${ }^{1}$ Neoliberal Policies and Early Childhood: a ReView From a Rights-Based APPROACH AND EDUCATIONAL INCLUSION IN CHILE
}

\author{
María Francisca MUÑOZ-OYARCE²
}

\begin{abstract}
RESUMEN: El artículo que a continuación se presenta es un ensayo, producto de una revisión documental, que aborda desde una perspectiva crítico-reflexiva la instalación de las políticas neoliberales y de mercado en la educación chilena, puesto que, desde su implantación con la dictadura militar sus consecuencias siguen vigentes, impactando al sistema en su conjunto. La educación es un proceso permanente que está siempre presente en la vida de los individuos, e influye en su forma de pensar, sentir y actuar, en consecuencia, la sociedad del tercer milenio demanda procesos educativos que tiendan a la consolidación de la calidad educativa, con fuerte énfasis en los principios de equidad y diversidad. En este sentido, el aula de primera infancia se enfrenta a una gran heterogeneidad social y cultural, que implica otra concepción de la organización en pre-escolar que va más allá del camino de la uniformidad y que reconoce la diferencia, considerando así la diversidad como un aspecto enriquecedor de la propia comunidad educativa. En el presente ensayo se analizan los límites y desafíos en la primera infancia, teniendo como telón de fondo el enfoque de derechos y los ingentes procesos de inclusión educativa, que procuran alcanzar una sociedad no sólo más justa y democrática, sino más respetuosa por la inclusión de todos los nińos(as), desde los primeros niveles educativos.
\end{abstract}

PALABRAS CLAVE: Políticas neoliberales. Primera infancia. Inclusión educativa. Enfoque de derechos.

\begin{abstract}
The article presented below is an essay, product of a documentary review, which addresses from a critical-reflective perspective the installation of neoliberal and market policies in Chilean education, since its implementation with the military dictatorship, its consequences remain in force, affecting the system as a whole. Education is a permanent process that is always present in the lives of individuals, and influences their way of thinking, feeling, and acting, consequently, the society of the third millennium demands educational processes that tend to the consolidation of educational quality, with a strong emphasis on the principles of equity and diversity. In this sense, the early childhood classroom faces great social and cultural heterogeneity, which implies another conception of the organization in preschool that goes beyond the path of uniformity and that recognizes difference, thus considering diversity as an enriching aspect of the educational community itself. In this essay, the limits and challenges in early childhood are analyzed, taking as a background the rights approach and the enormous processes of educational inclusion, which seek to achieve a society that is not only more just and democratic, but also more respectful of all children inclusion, from the earliest educational levels.
\end{abstract}

KEYWORDS: Neoliberal policies. Early childhood. Educational inclusion. Rights approach.

\section{INTRODUCCIÓN}

En Chile, la política neoliberal se instala de la mano de la dictadura militar ejercida por el general Augusto Pinochet y las fuerzas armadas desde septiembre del año 1973 hasta marzo del año 1990 (Aguilera, 2015). A partir de ello el modelo neoliberal expresa y sintetiza un ambicioso proyecto de reforma ideológica de la sociedad chilena, instalando una lógica de regulación de la vida a partir del libre comercio, invisibilizando varios de los derechos sociales fundamentales (Cavieres, 2009; Slachevsky, 2015). Esto ha tenido un impacto en todo orden, y el ámbito educativo no ha sido la excepción, toda vez que se tensiona fuertemente el concepto de educación como "bien de consumo" instalado en el marco del modelo vigente, frente a la

\footnotetext{
${ }^{1}$ https://doi.org/10.1590/1980-54702021v27e0039

${ }^{2}$ Magister en Educación Especial y Psicopedagogía. Estudiante programa Doctorado en Educación. Universidad Católica del Maule. Talca/Chile. E-mail: maria.munoz.19@alu.ucm.cl. ORCID: http://orcid.org/0000-0003-2027-2866
} 
educación como bien social que históricamente había sido la constante en el país (CamposMartinez et al., 2015; Donoso, 2005; Falabella, 2015). Así, la reforma abre sus puertas al dominio de profesionales de diversas áreas, especialmente del área económica, contribuyendo a la devaluación de la enseñanza por parte del cuerpo docente.

La retórica neoliberal asigna un papel estratégico a la educación y básicamente determina algunas acciones para conseguirlo de acuerdo a lo reportado por la literatura: En primer término, vincula la educación escolar a la preparación para el trabajo y la investigación académica al imperativo del mercado o a las necesidades de la empresa (Hill \& Kumar, 2012; Romé, 2016). De esta manera asegura que el mundo de los negocios tiene interés en la educación porque quiere una fuerza de trabajo calificada que sea capaz de competir en los mercados nacionales e internacionales. En este contexto, privilegia las técnicas de organización, el pensamiento de dimensión estratégica y la capacidad de trabajo en equipo. En segundo lugar, hace de la escuela un medio de transmisión de sus principios doctrinales, lo que no se cuestiona es la adaptación de la escuela a la ideología dominante, por tanto, la escuela tiene un papel importante en la difusión de la ideología oficial, en consecuencia, se pone un fuerte énfasis en el control para enmarcar la escuela, con el fin de cumplir más eficazmente su función de reproducción de la ideología dominante (Morley et al., 2014; Solimano, 2014).

En Chile, el Ministerio de Educación (MINEDUC) desde mediados de la década del 2000, se propuso como horizonte la construcción de una política pública pensada en los derechos de todos(as) los niños(as), en particular el derecho a una educación de calidad, que sea capaz de incluir y acoger en las aulas de jardines infantiles, escuelas y liceos a los niños(as) y jóvenes mayormente excluidos, no obstante, con acciones pensadas preferentemente en la educación especial (MINEDUC, 2005; 2009). De acuerdo a las recomendaciones entregadas por el informe de revisión, solicitado por el MINEDUC, llevado a cabo por la Organización

para la Cooperación y el Desarrollo Económicos (OCDE) se establece que es fundamental brindar oportunidades para que todos los "estudiantes chilenos(as), independiente de sus orígenes, desarrollen su máximo potencial de aprendizaje desde edades tempranas, desarrollando ambientes y prácticas que fortalezcan la calidad de dicho aprendizaje, bajo un paradigma inclusivo abordando tanto los procesos que este implique y sus estructuras" (OCDE, 2017 p. 17)

Para cumplir esta función de manera eficiente, es necesario volver a re-significar las políticas públicas en curso (MINEDUC, 2013; 2015). Lo anterior se ve reflejado en la promulgación de la ley 20.422 del año 2010, orientada a favorecer la igualdad de oportunidades e inclusión social de las personas que presentan discapacidad; la ley 20.609 del año 2012 que penaliza la discriminación arbitraria conocida como ley antidiscriminación; y seguidamente la ley 20.845 denominada ley de inclusión escolar que pone fin al lucro, la selección y el copago. A nivel de Educación parvularia se han llevado a cabo significativos avances en lo que a políticas públicas se refiere. El ańo 2015 se crea la Subsecretaría de educación parvularia, bajo la ley 20.835 en colaboración directa con el MINEDUC, siendo un órgano de colaboración, tanto en la organización general como en la promoción, desarrollo y coordinación de la calidad de la educación parvularia.

Desde otra perspectiva, la educación inclusiva ha surgido como un tema clave tanto en la investigación como en la práctica profesional, así como una cuestión apremiante en el 
programa de políticas educativas de los países. En los últimos años, la educación inclusiva ha recibido un impulso en muchos sistemas educativos (Amor et al., 2018; Ávila, 2019; PazMaldonado, 2020). Sobre la base de la crítica de la educación especial inspirada por la segregación de los estudiantes en condición de discapacidad, el debate científico se ha ampliado para converger hacia el concepto de educación inclusiva o educación para todos, promovidos a partir de la Conferencia de Salamanca (Organización de las Naciones Unidas para la Educación, la Ciencia y la Cultura [UNESCO], 1994). En la escuela, todos los nińos y jóvenes deben ser educados, independientemente de sus condiciones físicas, intelectuales, sociales, culturales, emocionales, lingüísticos u otros, a su vez, las diferencias individuales son conceptualizadas no como algo negativo que es necesario combatir, sino como un valor de referencia, ya que fundamentan y justifican cambios en la gestión del currículo de las escuelas y, consecuentemente, en el proceso de enseńanza y aprendizaje (UNESCO, 2008; 2015).

En este escenario, la primera infancia se constituye en una modalidad educativa fundamental para el desarrollo y adquisición de habilidades en diferentes áreas del desarrollo de los niños y niñas, pues es ahí donde se promueve el desarrollo físico, emocional, social e intelectual, así como su autoestima y confianza herramientas fundamentales que les permitirán superar las posibles dificultades que el proceso de alfabetización les impondrá (Finkelstein et al., 2019). De acuerdo a lo precedentemente expuesto, se pretende analizar como las políticas neoliberales y de mercado en la educación chilena, impactan el desarrollo de la primera infancia, con la progresiva instalación del enfoque de derechos y la perspectiva de inclusión educativa, que pretenden otorgan una educación equitativa y de calidad a todos los niños(as) del país.

\section{ENFOQUE DE DERECHOS: UNA PERSPECTIVA INTERNACIONAL}

Desde fines del siglo XIX con la realización del primer Congreso Internacional de Protección a la Infancia celebrado en París en el año 1883, del que posteriormente surgieron tres Congresos Internacionales de Gotas de Leche - París (1905); Bruselas, (1907) y Berlín (1911) de los cuales se concluye que los problemas sociales, la situación de la infancia, así como las implicancias políticas, traspasaban las fronteras, convirtiendo los problemas de infancia en un problema global (Dávila \& Naya, 2006). De esta forma se establecen' las bases para las posteriores políticas internacionales que surgen en favor de la infancia, al poner el énfasis en los nińos(as) abandonados, la exclusión social, la delincuencia o la tutela entre otros. Es así como el reconocimiento del niño(a) como sujeto de derechos ha consumido más de un siglo de discusión en la esfera internacional.

La situación crítica que vivió la infancia, en especial a principios de este siglo, agravada por el flagelo que representó la Primera Guerra Mundial, llevó a la Sociedad de Naciones, precursora de la actual ONU, a adoptar la primera Declaración de los Derechos del Niño en 1924, conocida como la Declaración de Ginebra (Liebel, 2009). Esta declaración, es un texto breve y genérico que consta de cinco artículos, representando un hito inicial para la promoción de los derechos en la primera infancia. Sentó las bases para el reconocimiento y la protección de los derechos de los nińos(as), así como para cristalizar los cambios en relación con el concepto de autonomía y los derechos de los niños(as) y los adolescentes. La Declaración de Ginebra 
también sacó a la luz el importante concepto del interés superior del niño(a), que posteriormente fue retomado y desarrollado por la Convención de 1989 (Romero, 2012).

Después de la Segunda Guerra Mundial, con la fundación de las Naciones Unidas, en 1947 se creó en su marco la UNICEF, el Fondo de las Naciones Unidas para la Infancia, un organismo especializado cuya misión fundamental es la defensa y promoción de los derechos del niño. Al año siguiente se aprobó la Declaración Universal de Derechos Humanos, cuya sección 25 trata específicamente de la protección de los niños. El 20 de noviembre de 1959, la Asamblea General de las Naciones Unidas adoptó una nueva Declaración de los Derechos del Niño. Se articula en diez principios que tratan de ampliar y actualizar la protección que ofrece la Declaración de Ginebra, además de incorporar nuevos parámetros de protección de los derechos humanos aplicables a los niños y los jóvenes (Liebel, 2009).

La comunidad internacional eligió 1979 como el Año Internacional del Niño(a). La repercusión del evento y la propuesta ofrecida previamente por la delegación polaca ante la ONU dieron inicio a las discusiones que, diez años después, llevarían a la firma de la Convención sobre los Derechos del Niño (UNICEF, 2006). La Convención sobre los Derechos del Niño, completó, mejoró y dio carácter vinculante a la Declaración aprobada por las Naciones Unidas treinta años antes. La ratificación de la Convención implica la revisión de la legislación nacional sobre los niños(as) y los jóvenes a fin de que se ajuste a las disposiciones establecidas en ella. Los derechos humanos nos obligan a tratar a los demás como a nosotros quisiéramos ser tratados, vale decir, con dignidad, respeto, igualdad y justicia y se aplican independientemente de la nacionalidad, raza, origen étnico, idioma, sexo, la orientación sexual, la capacidad, el origen social, entre otros. Sin embargo, los niños(as) también son diferentes de los adultos están en proceso de desarrollo y necesitan apoyo. Es por estas razones que tienen sus propios derechos. Con este fin, fue adoptada por las Naciones Unidas el 20 de noviembre de 1989, la Convención sobre los Derechos del Niño (CDN). La CDN es el tratado internacional más ampliamente aceptada hasta ahora, con el mayor número de Estados Partes, puesto que ha sido suscrito por 193 países (UNICEF, 2006).

La Convención se divide en algunos apartados: una primera parte con principios generales y 36 artículos en los que reconoce los derechos de los niños, niñas y adolescentes; una segunda parte con cuatro artículos en los que establece su órgano de control, el Comité de los Derechos del Niño; y una tercera parte con nueve artículos en los que establece los mecanismos de ratificación, adhesión, reservas y enmiendas (Llorens, 2012). A propósito de la perspectiva de educación e inclusión la Declaración explicita el siguiente artículo:

Artículo 2: Los Estados Partes respetarán los derechos enunciados en la presente Convención y asegurarán su aplicación a cada niño(a) sujeto a su jurisdicción, sin distinción alguna, independientemente de la raza, el color, el sexo, el idioma, la religión, la opinión política o de otra índole, el origen nacional, étnico o social, la posición económica, los impedimentos físicos, el nacimiento o cualquier otra condición del niño(a), de sus padres o de sus representantes legales.

En este sentido, los derechos del niño(a) se consideran como un conjunto de valores consagrados en instrumentos jurídicos internacionales o nacionales, que tienen por objeto garantizar el respeto y la puesta en práctica de determinados principios y condiciones de vida de los nińos(as) (Vanegas, 2017). Aunque se trata de un tema cuya importancia es inestimable, 
seguimos siendo testigos de la casi total ignorancia de los adultos sobre lo que son y lo que implican. Muchos educadores y profesores no sólo desconocen su existencia real, sino también, y lo que es más importante, que los derechos pueden ser la guía de su acción educativa y pedagógica (Ortiz, 2009).

La educación en la primera infancia forma parte del derecho a la educación, universalmente garantizada en los instrumentos del derecho internacional y los derechos humanos. Estos instrumentos internacionales tienen dos aspectos comunes: primero, el reconocimiento, explícito que el aprendizaje comienza con el nacimiento; en segundo lugar, el reconocimiento de que la educación y el cuidado de la primera infancia no son dimensiones distintas o incoherentes (UNICEF, 2006). En este sentido, la subordinación de políticas de educación a las necesidades del mercado y la falta de voluntad política por parte de los gobiernos, ha conducido a que la perspectiva de la educación de la primera infancia como derecho humano, haya sido suplantada en gran medida por una visión economicista y utilitaria de la educación, basado en la idea de crear capital humano. Sin embargo, el concepto de educación y cuidado de la primera infancia, cuando se basa en la perspectiva de derechos humanos, no sólo tiene la posibilidad de preparar a las personas para sus vidas futuras y para construir mejores condiciones económicas, sino que considera la promoción de la igualdad, la superación de la pobreza y la equidad social (Bakken et al., 2017; Sounoglou \& Michalopoulou, 2017). Una visión reductora de la educación en la primera infancia, minimiza el papel de los actores principales - los niños y las niñas - y les impide ejercer una participación activa, valorando su futuro y no su presente, alimentando así grandes asimetrías estructurales, que generalmente están en la raíz de la exclusión y discriminación.

\section{Derechos y PRimera infancia EN Chile}

La CDN es ratificada por Chile el 14 de agosto del año 1990, vale decir, sólo un año más tarde de su promulgación a nivel internacional. Desde ese momento son diversas las iniciativas que procuran instalar la perspectiva de derechos en la infancia, en un país que se abre a la democracia luego de 17 ańos de dictadura. La suscripción por Chile de la CDN logra un paso importante en la concepción de infancia, toda vez que a partir de ahora los niños y niñas son vistos como sujetos de derechos, cuestión que permitiría re-direccionar las políticas públicas desde una perspectiva de asistencialidad, a una mirada que comprenda el desarrollo infantil desde una óptica asociada al desarrollo humano integral. No obstante, la literatura reporta, que los avances en términos del reconocimiento de los niños y niñas durante la década del 90, fueron más bien escasos desde el plano institucional y jurídico, mantenido la lógica de apoyo a los grupos mayormente vulnerados (Torres, 2008). Iniciando la década del 2000, surge la Política Nacional de la Infancia y Adolescencia y su Plan de Acción Integrado 2001 - 2010, esta política se configura como un marco para todas aquellas iniciativas que se dirigen hacia la protección de la infancia. En la misma década surge el programa Chile Crece Contigo, este Sistema busca generar, potenciar y articular todas las políticas e iniciativas públicas que apoyen el desarrollo de niños y niñas (Ochoa et al., 2010).

A partir del año 2010, en Chile vuelve con fuerza la concepción de la educación como un derecho social, cuestión que lleva a cambios significativos en la política pública educativa. Es en este contexto, que se levanta el texto Enfoque de derechos aplicados en la Educación 
Parvularia, documento técnico elaborado por la Superintendencia de Educación, específicamente la Intendencia de Educación Parvularia el ańo 2018, y tiene como propósito instalar la perspectiva de los derechos humanos en la educación infantil (MINEDUC, 2018). El diseño, implementación y ejecución de las políticas, planes y programas relacionados con la infancia, se orientan hacia una perspectiva de "enfoque de derechos", en contraposición al "enfoque de necesidad" que primaba con anterioridad a la entrada en vigencia de la CDN, conforme al cual al Estado le cabía sólo un rol de asistencia en materia de primera infancia. Lo anterior, en el bien entendido que los niños(as) son portadores de todas las garantías que consagra y define la CDN, y consecuentemente, tienen derecho a medidas especiales de protección y al ejercicio progresivo de sus derechos (Miranda Valdebenito \& González Burboa, 2016). En este sentido, se considera de suma relevancia el respeto de los intereses, experiencias y problemas que afrontan los niños(as) de 0 a 6 ańos, eliminando cualquier signo de exclusión o discriminación en términos de políticas y prácticas pedagógicas.

\subsection{INCLUSIÓN EDUCATIVA EN PRIMERA INFANCIA}

La inclusión se refiere básicamente a las cuestiones de derechos humanos, igualdad, justicia social y la lucha por una sociedad igualitaria y no discriminatoria (Blanco \& Duk, 2019). Estos temas son el núcleo de las políticas y prácticas inclusivas. La inclusión representa un proceso, una filosofía de aceptación, la capacidad de proporcionar un marco en el que los estudiantes, independientemente de sus capacidades, género, idioma, origen étnico o cultural, puedan ser valorados por igual, tratados con respeto y se les ofrezcan las mismas oportunidades que a los demás estudiantes de la escuela (Ainscow, 2017; Kassar, 2016; Marchesi \& Hernández, 2019). El concepto central del "principio de inclusión" es que todos(as) los estudiantes son únicos, con sus experiencias, predilecciones y actitudes, y corresponde a la escuela adaptarse al estudiante, para aprovechar la diversidad existente (Armijo-Cabrera, 2018; Petriwskyj, 2010). Analizar el concepto de inclusión requiere asumir que todos los estudiantes aprenden juntos, en relación con las diferencias ambientales, físicas y socio-afectivas, lo que representa numerosas ventajas. En este contexto, el concepto de inclusión se basa no sólo en la medición de la distancia entre el supuesto estándar de adecuación, sino también en el reconocimiento de la relevancia de la plena participación en la vida escolar por parte de todos los sujetos (Echeíta, 2008; Polat, 2011).

El término "educación inclusiva", se generalizó a través de la Declaración de Salamanca (UNESCO, 1994), desde entonces en la literatura educativa asumió el concepto de "escuela para todos", en referencia al conjunto de estudiantes que vienen siendo tradicionalmente marginados por la escuela, considerados en términos genéricos como estudiantes con necesidades educativas especiales. A pesar del aparente consenso sobre el principio filosófico de la inclusión escolar, difícilmente se encuentra en la literatura una definición precisa para ese término, pero el atributo principal ha sido la garantía de plena participación de todos los alumnos(as), especialmente aquellos que se encuentran en riesgo de exclusión, vale decir, rescatar el principio de la diversidad para instalarlo en la escuela desde un enfoque que favorece y privilegia la inclusión en las aulas (Rojas \& Armijo, 2016). Lo anterior, puesto que la noción de inclusión considera no sólo a las personas con discapacidad, sino que más bien a quienes han sido históricamente excluidos de los procesos sociales y educativos, como aquellos que viven en 
la pobreza, o presentan una etnia, orientación sexual, expresión de género o religión diferente, vale decir, la inclusión lleva implícita el respeto a la diversidad como una condición de la sociedad contemporánea, caracterizada por entornos educativos complejos (UNESCO, 2020).

La educación infantil contribuye de forma significativa al desarrollo físico, psicológico, intelectual y social de los niños y niñas. En este sentido, la educación preescolar debe proporcionar experiencias e interacciones con el mundo social y físico, de forma ajustada a las sucesivas edades que abarca y siguiendo los principios pedagógicos, de acuerdo con el desarrollo de cada niño(a) (Bakken et al., 2017). El reconocimiento de la educación infantil es un deber del Estado, que debe velar por su cumplimiento y puesta en vigencia en el territorio nacional (Díaz, 2018). En este contexto, todos los niños(as) del país, han asegurado por las políticas educativas vigentes, el derecho a una educación pública y de calidad. Sin embargo, surgen diversos obstáculos para la materialización de ese derecho, tales como: precarización de la formación docente para la actuación en la perspectiva de la educación inclusiva; falta de infraestructura de los sistemas educativos y de las escuelas en general y de los jardines infantiles en particular, que a menudo presentan diferentes barreras al acceso, permanencia y participación y en consecuencia no se encuentran preparadas para atender la diversidad de los estudiantes (Aincow, 2017; Vergara, 2019).

\section{Propuestas y reflexiones posibles}

En el contexto de lo precedentemente expuesto, podríamos afirmar que efectivamente el Estado de Chile considera la perspectiva de derechos en la educación de niños y niñas de 0 a 6 años. No obstante, y a propósito de los aspectos considerados en la introducción de este ensayo cabe la pregunta ¿es posible que un Estado que promueve un modelo neoliberal en educación, implemente efectivamente las políticas internacionales referidas a los derechos del niño y la niña? Para responder a lo anterior, tendríamos al menos dos miradas con respecto al papel del Estado en educación, puesto que, desde la perspectiva del Estado de bienestar, el papel de la educación es muy claro: el Estado se concibe como aquel que tiene como principio la garantía del derecho a la ciudadanía y, por lo tanto, busca incluir a todos los sujetos. El Estado se concibe como el que debe intermediar en las negociaciones entre los sujetos y actuar como regulador y promotor de alguna forma de participación de los excluidos del proceso (Heckman, 2008). Por otro lado, en un Estado Neoliberal, las reglas son diferentes. En primer lugar, el sujeto no es un ciudadano, sino un consumidor, es decir, su potencial de consumo es establecer su lugar social (Huerta-Moreno, 2005; Villalobos \& Quaresma, 2015).

Actualmente, el neoliberalismo en su búsqueda de hegemonía en el proceso productivo mundial, trata de entrar en la educación a través de un nuevo modelo de pedagogía instalado desde los primeros años de escolaridad (Aguilera, 2015). En consecuencia, resulta altamente complejo pretender que sólo por el hecho de estipular o incluir la perspectiva de derechos en el currículo de la educación parvularia, el Estado asuma un compromiso distinto con su formación, puesto que implícitamente Chile ha privilegiado no sólo el discurso neoliberal, sino una práctica educativa, que conduce a la segregación, la competencia y la exclusión social de aquellos que se encuentran mayormente marginados socialmente (Campos-Martinez et al., 2015; Donoso, 2005). 
En la actualidad se comprende a la educación parvularia como una instancia que hace cumplir los derechos de los niños(as), brindándoles el derecho a una educación de calidad, a estar en un ambiente acogedor y seguro, a una alimentación sana, con el derecho a la higiene y al juego, entre otros derechos, comprendiendo sus necesidades y las de su familia, En consecuencia, esta mirada requiere no sólo una reforma educacional, sino más bien una reforma político-social que conciba al Estado en un rol social y no subsidiario como actualmente lo tenemos en Chile (Huerta-Moreno, 2005). Por tanto, de no mediar lo anterior, resulta difícil y complejo asumir a los niños y nińas como sujetos de derecho desde la primera infancia. En línea con lo expuesto, surge una nueva interrogante ¡es posible que el sistema neoliberal genere cambios que vayan en la dirección de una mayor inclusión, justicia y equidad social desde la primera infancia? Diversos autores Cavieres (2009), Donoso (2005), Falabella (2015), Rivero-Bottero (2013), Villalobos y Quaresma (2015) seńalan que el sistema capitalista de mercado genera pobreza y miseria, en el que la igualdad de oportunidades para todos es una falacia que sostiene una profunda desigualdad social y educativa, por tanto, se convierte en un desafío ético discutir los derechos de los niños(as) en una sociedad que, con sus diversas leyes y documentos, los afirma, pero en la práctica no cumple. Por tanto, independientemente del género, la clase social u otras características individuales y/o sociales, la inclusión es un derecho fundamental de todos los niños y niñas y, en consecuencia, el derecho a la inclusión no puede negarse a ningún grupo social o de edad (Armijo-Cabrera, 2018; Blanco \& Duk, 2019). En este sentido podemos afirmar que la perspectiva de inclusión educativa en primera infancia resulta atractiva como tema, sin embargo, funciona más como un eslogan y muy raramente se traduce en acciones continuas, integradas, transversales y significativas para los niños y niñas, en orden a sostener una mayor equidad o justicia social.

\section{Conclusiones}

Hablar de ideología neoliberal y educación es una empresa muy compleja, puesto que ambos temas son casi inagotables. En general, existe una tendencia a oponer la educación como formación y la educación como información, oposición que reaparece cuando se distingue entre aprendizaje y formación, espíritu crítico y automatización. En este sentido, la propuesta es hacer que la educación pueda ser una posibilidad de conocimiento y transformación gracias a su comprensión crítica, que permita re-mirar el fenómeno de la racionalidad o ideología neoliberal a objeto de comprender esa realidad, pero a su vez transformarla, transitando desde una visión tecnocrática hacia una perspectiva humanista de la educación. En esta línea podemos afirmar que mantener el sistema educativo bajo el actual modelo político, inevitablemente trae consecuencias, desde una dimensión técnica, tensiona a la educación parvularia a regular su labor educativa por medio de la medición, estandarización y objetivación del proceso de enseńanza, enfatizando una visión productiva, la cual estratifica los jardines infantiles y en consecuencia a sus profesionales, según estándares de calidad, los cuales luego impactan en un sistema educativo pensado desde y para el mercado, como un lugar donde confluyen la oferta y demanda de bienes y servicios.

Desde otra perspectiva, podemos señalar que la educación inclusiva ha sido cada vez más valorada, tanto en Chile como en el resto del mundo. Hay varios agentes involucrados en la educación preescolar: padres, otros parientes, educadoras, políticos, psicólogos, sociólogos 
y la propia comunidad. Todos estos actores contribuyen al desarrollo del proceso educativo de cada niño(a), que es inagotable y continuo, es decir, la educación es un proceso permanente que está siempre presente en la vida de los individuos, que influye en su forma de pensar y actuar. En este sentido el aula de primera infancia se enfrenta a una gran heterogeneidad social y cultural, que implica otra concepción de la organización en pre-escolar que va más allá del camino de la uniformidad y que reconoce la diferencia, considerando así la diversidad como un aspecto enriquecedor de la propia comunidad educativa.

En este contexto, se considera de suma relevancia el respeto de los intereses, experiencias y problemas que afrontan los niños(as) de 0 a 6 años y en este sentido no debe existir ningún signo de exclusión o discriminación en términos de políticas y prácticas pedagógicas, muy por el contrario, se debe desplegar las acciones necesarias que favorezcan el pleno desarrollo de los nińos y niñas desde una perspectiva de inclusión educativa. En esta línea, se propone reflexionar sobre la respuesta que la educación parvularia ofrece a los niños(as), en el bien entendido que ellos tienen derecho a una educación de calidad donde puedan obtener respuestas para sus necesidades, socializar con otros niños(as), aprender junto a ellos, para vivir con la comunidad educativa y la sociedad de la que forman parte.

\section{REFERENCIAS}

Aguilera, N. (2015). Una revolución neoliberal: la política educacional en Chile desde la dictadura militar. Educação e Pesquisa, 41(special), 1473-1486. http://dx.doi.org/10.1590/S15179702201508141660

Ainscow, M. (2017). Haciendo que las escuelas sean más inclusivas: lecciones a partir del análisis de la investigación internacional. Revista de Educación Inclusiva, 5(1), 39-49. https:// revistaeducacioninclusiva.es/index.php/REI/article/viewFile/220/214

Amor, A., Hagiwara, M., Shogren, K., Thompson, J., Verdugo, M., Burke, K., \& Aguayo, V. (2018). International perspectives and trends in research on inclusive education: a systematic review. International Journal of Inclusive Education, 23(12), 1277-1295. https://doi.org/10.1080/13603 116.2018.1445304

Armijo-Cabrera, M. (2018). Deconstruyendo la noción de inclusión: un análisis de investigaciones, políticas y prácticas en educación. Revista Electrónica Educare, 22(3), 151-176. http://dx.doi. org/10.15359/ree.22-3.8

Ávila, M. (2019). Inclusión del Siglo XXI: reflexiones sobre la Educación Inclusiva en Chile y Latinoamérica. Revista de Educación Inclusiva/Polyphōnía, 3(2), 153-185. https://core.ac.uk/ download/pdf/225618181.pdf

Bakken, L., Brown, N., \& Downing, B. (2017). Early childhood education: The long-term benefits. Journal of Research in Childhood Education, 31(2), 255-269. https://doi.org/10.1080/02568543.2 016.1273285

Blanco, R., \& Duk, C. (2019). El legado de la Conferencia de Salamanca en el pensamiento, políticas y prácticas de la educación inclusiva. Revista latinoamericana de educación inclusiva, 13(2), 25-43. http://dx.doi.org/10.4067/S0718-73782019000200025

Campos-Martinez, J., Corbalán, F., \& Inzunza, J. (2015). Mapping neoliberal reform in Chile. Mapping Corporate Education Reform: Power and Policy Networks in the Neoliberal State. Routledge. 
Cavieres, E. (2009). La neoliberalización de la educación chilena o la privatización del sistema educativo por dentro. Diálogos Educativos, 9(17), 18-57. https://www.researchgate.net/publication/28319294_La_ neoliberalizacion_de_la_educacion_chilena_o_la_privatizacion_del_sistema_educativo_por_dentro

Dávila, P., \& Naya, L. (2016). La evolución de los derechos de la infancia: Una visión internacional. Encounters in Theory and History of Education, 7. https://doi.org/10.24908/eoe-ese-rse.v7i0.597

Díaz, M. (2018). Reformas curriculares de educación infantil temprana el caso de Chile. UNESCO - OIE. http://148.102.58.24/handle/MINEDU/5886.

Donoso, S. (2005). Reforma y política educacional en Chile 1990-2004: El neoliberalismo en crisis. Estudios Pedagógicos, 31(1), 113-135. http://dx.doi.org/10.4067/S0718-07052005000100007

Echeíta, G. (2008). Inclusión y exclusión educativa. "Voz y quebranto”. Revista Electrónica Iberoamericana sobre Calidad, Eficacia y Cambio en Educación, 6(2), 9-18, https://www.redalyc.org/articulo. oa?id $=551 / 55160202$

Falabella, A. (2015). El mercado escolar en Chile y el surgimiento de la Nueva Gestión Pública: El tejido de la política entre la dictadura neoliberal y los gobiernos de la centroizquierda (1979 a 2009). Educaçâo \& Sociedade, 36(132), 699-722. https://doi.org/10.1590/ES0101-73302015152420

Finkelstein, S., Sharma, U., \& Furlonger, B. (2019). The inclusive practices of classroom teachers: a scoping review and thematic analysis. International Journal of Inclusive Education, 1-28. https://doi. org/10.1080/13603116.2019.1572232

Fondo de las Naciones Unidas para la Infancia. (2006). Convención sobre los Derechos del Niño. UNICEF. https://www.un.org/es/events/childrenday/pdf/derechos.pdf

Heckman, J. (2008). La viabilidad del Estado del Bienestar. Cuadernos de Pensamiento Político, 20, 135171. https://www.jstor.org/stable/25597165?seq=1

Hill, D., \& Kumar, R. (2012). Global neoliberalism and education and its consequences. Routledge.

Huerta-Moreno, M. (2005). El neoliberalismo y la conformación del Estado subsidiario. Politica y cultura, 24, 121-150. http://www.scielo.org.mx/scielo.php?script=sci_ arttext\&pid=S0188-77422005000200006

Kassar, M. (2016). Escola como espaço para a diversidade e o desenvolvimento humano. Educação \& Sociedade, 37(137), 1223-1240. http://dx.doi.org/10.1590/es0101-73302016157049

Ley 20.422. (2010). Establece normas sobre igualdad de oportunidades e inclusión social de personas con discapacidad. https://www.leychile.cl/Navegar?idLey=20422

Ley 20.609. (2012). Establece medidas contra la discriminación. https://www.leychile.cl/ Navegar?idNorma $=1042092$

Ley 20.835. (2015). Crea la Subsecretaría de Educación Parvularia, la Intendencia de Educación Parvularia, y modifica diversos cuerpos legales. https://www.bcn.cl/leychile/navegar?idNorma=1077041

Ley 20.845. (2015). Inclusión escolar que regula la admisión de los y las estudiantes, elimina el financiamiento compartido y prohíbe el lucro en establecimientos educacionales que reciben aportes del estado. https://www.leychile.cl/Navegar?idNorma $=1078172$

Liebel, M. (2009). Sobre la historia de los Derechos de la infancia. Infancia y derechos humanos: hacia una ciudadanía participante y protagónica. IFEJANT, 23-40. http://de0a18.net/pdf/doc_participacio_ infancia_derechos_humanos.pdf\#page=23 
LLorens, J. (2012). La Convención sobre los Derechos del Niño: significado, alcance y nuevos retos. Educatio Siglo XXI, 30(2), 47-68. https://revistas.um.es/educatio/article/view/153681

Marchesi, Á., \& Hernández, L. (2019). Cinco Dimensiones Claves para Avanzar en la Inclusión Educativa en Latinoamérica. Revista latinoamericana de educación inclusiva, 13(2), 45-56. http:// dx.doi.org/10.4067/S0718-73782019000200045

Ministerio de Educación. (2005). Política Nacional de educación especial. http://centroderecursos. educarchile.cl/bitstream/handle/20.500.12246/2947/POLiTICAEDUCESP.pdf?sequence=1

Ministerio de Educación. (2009). Fija normas para determinar los alumnos con necesidades educativas especiales que serán beneficiarios de las subvenciones para educación especial., Decreto 170 C.F.R. https://www.bcn.cl/leychile/navegar?idNorma=1012570

Ministerio de Educación. (2013). Orientaciones técnicas para los Programas de Integración Escolar (PIE). https://especial.mineduc.cl/implementacion-dcto-supr-no170/orientaciones/

Ministerio de Educación. (2015). Propuestas para avanzar hacia un sistema educativo inclusivo en Chile: Un aporte de la Educación Especial. MINEDUC.

Ministerio de Educación. (2018). Enfoque de derechos en la Educación Parvularia. Santiago. Intendencia de Educación Parvularia. https://educrea.cl/enfoque-de-derechos-en-la-educacion-parvularia/

Miranda Valdebenito, N., \& González Burboa, A. (2016). El enfoque de derecho de la infancia y adolescencia en el contexto chileno. Humanidades Médicas, 16(3), 459-474. http://scielo.sld.cu/ scielo.php?pid=S1727-

Morley, L., Marginson, S., \& Blackmore, J. (2014). Education and neoliberal globalization. British Journal of Sociology of Education, 35(3), 457-468. https://doi.org/10.1080/01425692.2014.893072

Ochoa, G., Maillard, C., \& Solar, X. (2010). Primera infancia y politicas públicas, una aproximación al caso del Sistema Integral de Protección a la Infancia Chile Crece Contigo. https://germina.cl/wpcontent/ uploads/2011/05/publicacion4_infancia_politicas_publicas_germina2010.pdf

Organización de las Naciones Unidas para la Educación, la Ciencia y la Cultura. (1994). Declaración de Salamanca: Sobre Principios, Politicas y Prácticas en el Área de las Necesidades Educativas Especiales. Salamanca, España.

Organización de las Naciones Unidas para la Educación, la Ciencia y la Cultura. (2008). Conferencia Internacional de Educación "la Educación Inclusiva: el Camino hacia el Futuro". Ginebra.

Organización de las Naciones Unidas para la Educación, la Ciencia y la Cultura. (2015). Incheon declaration. Education 2030: Towards inclusive and equitable quality education for all. Paris.

Organización de las Naciones Unidas para la Educación, la Ciencia y la Cultura. (2020). Relatório "Inclusão e educação: Todos, sem exceção". Paris.

Organización para la Cooperación y el Desarrollo Económicos. (2017). Education at a Glance 2017: OECD Indicators. OECD Publishing.

Ortiz, L. (2009). La Convención de los Derechos del Niño veinte ańos después. Revista Latinoamericana de Ciencias Sociales, Niñez y Juventud, 7(2), 587-619. http://ns520666.ip-158-69-118.net/rlcsnj/ index.php/Revista-Latinoamericana/article/view/186

Paz-Maldonado, E. (2020). Una Aproximación Teórica sobre la Educación Inclusiva en Honduras: Avances, Obstáculos y Desafíos. Revista Brasileira de Educação Especial, 26(3), 371-386. https:// doi.org/10.1590/1980-54702020v26e0049 
Petriwskyj, A. (2010). Diversity and inclusion in the early years. International Journal of Inclusive Education, 14, 195-212. https://doi.org/10.1080/13603110802504515

Polat, F. (2011). Inclusion in education: A step towards social justice. International Journal of Educational Development, 31(1), 50-58. http://dx.doi.org/10.1016/j.ijedudev.2010.06.009

Rivero-Bottero, R. (2013). Educación y Pedagogía en el marco del neoliberalismo y la globalización: Perfiles Educativos, 35(142), 149-166. http://www.scielo.org.mx/scielo.php?script=sci_ arttext\&pid=S0185-26982013000400010

Rojas, M. T., \& Armijo, M. (2016). Qué es la inclusión escolar: distintas perspectivas en debate. Cuadernos de Educación, 75, 1-11. https://www.uahurtado.cl/cuadernos_educacion_75/

Romé, N. (2016). El presente totalitario de la ideología neoliberal. Utopía y Praxis Latinoamericana, 21(74), 99-110. https://www.redalyc.org/articulo.oa?id=279/27950106009

Romero, B. (2012). Derechos de la Infancia y Educación Inclusiva en América Latina. Revista Española de Educación Comparada, 20, 401-407. https://search.proquest.com/docview/1418692407/ fulltextPDF/2E7E67C148BB4E77PQ/1?accountid=170518

Slachevsky, N. (2015). Una revolución neoliberal: la política educacional en Chile desde la dictadura militar. Educação e Pesquisa, 41(special), 1473-1486. http://dx.doi.org/10.1590/S15179702201508141660

Solimano, A. (2014). Chile and the neoliberal trap. Cambridge Books.

Sounoglou, M., \& Michalopoulou, A. (2017). Early Childhood Education Curricula: Human Rights and Citizenship in Early Childhood Education. Journal of Education and learning, 6(2), 53-68. http://dx.doi.org/10.5539/jel.v6n2p53

Torres, O. (2008). Niñez, políticas públicas y sociedad civil. Revista MAD Edición especial. 3, 17-45. http://repositorio.uchile.cl/handle/2250/121621

Vanegas, B. (2017). La infancia como sujeto de derechos. Un análisis crítico. Revista Ratio Juris, 12(24), 127-142. https://dialnet.unirioja.es/servlet/articulo?codigo=6748976

Vergara, F. (2019). Inclusión educativa en Chile: un camino político-histórico con una ruta de empedrados, curvas y colinas. Revista Estudios en Educación, 2(2), 143-167. http://ojs.umc.cl/ index.php/estudioseneducacion/article/view/53

Villalobos, C., \& Quaresma, M. (2015). Sistema escolar chileno: características y consecuencias de un modelo orientado al mercado. Convergencia, 22(69), 63-84. http://www.scielo.org.mx/scielo. php?pid=S140514352015000300063\&script=sci_arttext ${ }^{-}$

Recebido em: 09/03/2021

Reformulado em: 10/06/2021

Aprovado em: 20/07/2021 